Article

\title{
Optimal Dividend Payment in De Finetti Models: Survey and New Results and Strategies
}

\author{
Christian Hipp
}

Karlsruhe Institute of Technology, D-51465 Bergisch Gladbach, Germany; christian.hipp@kit.edu; Tel.: +49-2202-188-2031

Received: 27 July 2020; Accepted: 7 September 2020; Published: 10 September 2020

Abstract: We consider optimal dividend payment under the constraint that the with-dividend ruin probability does not exceed a given value $\alpha$. This is done in most simple discrete De Finetti models. We characterize the value function $V(s, \alpha)$ for initial surplus $s$ of this problem, characterize the corresponding optimal dividend strategies, and present an algorithm for its computation. In an earlier solution to this problem, a Hamilton-Jacobi-Bellman equation for $V(s, \alpha)$ can be found which leads to its representation as the limit of a monotone iteration scheme. However, this scheme is too complex for numerical computations. Here, we introduce the class of two-barrier dividend strategies with the following property: when dividends are paid above a barrier $B$, i.e., a dividend of size 1 is paid when reaching $B+1$ from $B$, then we repeat this dividend payment until reaching a limit $L$ for some $0 \leq L \leq B$. For these strategies we obtain explicit formulas for ruin probabilities and present values of dividend payments, as well as simplifications of the above iteration scheme. The results of numerical experiments show that the values $V(s, \alpha)$ obtained in earlier work can be improved, they are suboptimal.

Keywords: stochastic control; optimal dividend payment; ruin probability constraint, De Finetti model

\section{Introduction}

We consider the computation of optimal dividend payment under the constraint that the ruin probability with possible dividend payment does not exceed a given value $\alpha .{ }^{1}$ This is done in most simple De Finetti models with risk process

$$
S(t)=s+\sum_{i=1}^{t} X_{i}
$$

where $X_{1}, X_{2}, \ldots$ are independent with $\mathbb{P}\left\{X_{i}=1\right\}=p, \mathbb{P}\left\{X_{i}=-1\right\}=1-p, 1 / 2<p<1, q=1-p$, and initial surplus $s \geq 0$, which are discrete in time and space, are stationary, have independent increments, and are skip free. Admissible dividend strategies are integer valued functions $\delta(t) \geq 0, t \geq 0$, depending on $X_{1}, \ldots, X_{t}$; the with-dividend process

$$
S^{\delta}(t)=s+\sum_{i=1}^{t} X_{i}-\delta(i)
$$

\footnotetext{
1 Dedicated to David Dickson, Professor emeritus of The University of Melbourne
} 
has ruin time $\tau=\tau(s, \delta)=\inf \left\{t: S^{\delta}(t)<0\right\}$. Clearly, no dividends are paid at or after ruin. The present value of dividend payments with dividend strategy $\delta(t)$ and initial surplus $s$ is

$$
U(s, \delta)=E\left[\sum_{t=0}^{\tau-1} r^{t} \delta(t)\right],
$$

and the maximal possible present value $U(s)$ is also called value of the company.

This concept was introduced by DeFinetti (1957) for companies with risky business; he proved that the optimal dividend strategy is a barrier strategy with constant barrier $K$, and

$$
U(s)=w(s) /(w(K+1)-w(K)),
$$

where $w(s)$ is the score function discussed below, and $K$ is the minimizer for $w(s+1)-w(s)$. This produces a with-dividend risk process which is bounded by $K$ and so has ruin probability 1 . Gerber (1969) solved the optimal dividend problem for compound Poisson processes; he showed that band strategies are optimal. Also for these, the with-dividend process is bounded and so has ruin probability 1 . The unconstrained optimal dividend problem was investigated for many different model classes: for diffusion processes (see Asmussen and Taksar (1997)), for diffusion models with regime switching (see Sotomayor and Cadenillas (2011)), for jump-diffusion processes (see Belhaj (2010)), and even for spectrally negative Lévy processes (see Loeffen (2008) with a surprisingly simple condition for optimality of barrier strategies). Azcue and Muler (2005) present a compound Poisson example with Erlang claimsize distribution for which the optimal dividend strategy is not a barrier strategy (section 10.1 on p. 274). Optimal dividend problems are often solved via Hamilton-Jacobi-Bellman equations and their viscosity solutions. A survey on recent results for optimal dividend payment in collective risk models is Albrecher and Thonhauser (2009), while Schmidli's book (Schmidli 2007) is a more elementary introduction to these control problems for discrete models (chapter 1.2) as well as continuous models (chapters 2.4 and 2.5).

Several modifications of dividend payment were considered to avoid certain ruin. One is the use of non-constant linear or nonlinear dividend barriers (see Gerber (1981) and Albrecher and Kainhofer (2002)), a second is the concept of capital injections (see Kulenko and Schmidli (2008)).

Here we include the ruin probability into the optimization problem. The company value with ruin constraint $V(s, \alpha)$ is the maximal present value of dividend strategies $\delta(t)$ with a with dividend ruin probability

$$
\psi_{\delta}(s)=\mathbb{P}\{\tau(s, \delta)<\infty\}
$$

not exceeding $\alpha$, where $\alpha$ is given by the financial supervisor or other stakeholders. Clearly, $V(s, \alpha)=0$ for $s=-1$ and all $\alpha$, or for $\alpha \leq \psi_{0}(s)$ and all $s \geq 0$, where $\psi_{0}(s)$ is the ruin probability without dividend payments. Motivation for this problem can be found in Dickson and Drekic (2008) as well as in Hipp (2018) and Hipp (2019). Our aim is to characterize and compute the value function $V(s, \alpha)$ of this problem, and to compute the corresponding dividend strategies.

An early solution to the problem can be found in Hipp (2003); there, the following modified Hamilton-Jacobi-Bellman equation for the value function $V(s, \alpha)$ is presented:

$$
V(s, \alpha)=\sup _{\delta, \beta_{1}, \beta_{2}}\left\{\delta+r p V\left(s+1-\delta, \beta_{1}\right)+r q V\left(s-1-\delta, \beta_{2}\right)\right\}, s \geq 0,
$$

where the supremum is taken over all $0 \leq \delta \leq s$ and $\beta_{1}, \beta_{2}$ satisfying the admissibility condition

$$
\begin{aligned}
\psi_{0}(s+1-\delta) & \leq \beta_{1} \leq 1, \\
\psi_{0}(s-1-\delta) & \leq \beta_{2} \leq 1, \\
p \beta_{1}+q \beta_{2} & =\alpha .
\end{aligned}
$$


In Hipp (2003) the above Bellman equation was solved via the following iteration scheme: with an initial solution $V_{0}(s, \alpha), s \geq 0,0 \leq \alpha \leq 1$ we define $V_{n}(s, \alpha)$ recursively as

$$
V_{n+1}(s, \alpha)=\sup _{\delta, \beta_{1}, \beta_{2}}\left\{\delta+r p V_{n}\left(s+1-\delta, \beta_{1}\right)+r q V_{n}\left(s-1-\delta, \beta_{2}\right)\right\}, s \geq 0 .
$$

The intuition behind this simple recursion is the following: with initial state $(s, \alpha)$ at time $t \geq 0$ we pay out an amount $\delta$ as dividends and continue with state $s-\delta$. At time $t+1$ we are in state $s-\delta-1$ with probability $q$ and in state $s-\delta+1$ with probability $p$. We select probabilities $\beta_{1}, \beta_{2}$ which are admissible and maximize the expected present value of dividend payments after time $t$. The corresponding dividend strategies used after time $t$ are optimal for the states $\left(s-\delta+1, \beta_{1}\right)$ and $\left(s-\delta-1, \beta_{2}\right)$, respectively.

In Hipp (2003) it is also shown that (1) has exactly one solution $V(s, \alpha)$ which is the present value of an optimal dividend payment function $\delta(t, s, \alpha), t \geq 0$, for initial surplus $s$ and allowed ruin probability $\alpha$. An optimal dividend strategy can be obtained from (1):

$$
\delta(t, s, \alpha)=d\left(S^{\delta}(t), \alpha(t)\right),
$$

where $\alpha(t)$ is the running allowed ruin probability, with $d(s, \alpha)=k$ and $\alpha(t)$ is defined sequentially as

$$
\begin{aligned}
\alpha(0) & =\alpha, \\
\alpha(t+1) & =\beta_{1} \text { if } X_{t+1}=1 \\
\alpha(t+1) & =\beta_{2} \text { if } X_{t+1}=-1
\end{aligned}
$$

with $\left(k,\left(\beta_{1}, \beta_{2}\right)\right)$ the maximizer in the supremum in (1) for $V(s, \alpha(t))$, and $t \geq 0$.

If the suprema in (1) for $\delta=0$ and $\delta=1$ are equal, then the choices $d(s, \alpha)=0$ and $d(s, \alpha)=1$ are both optimal, they result in the same present value of dividend payments and the same ruin probability.

In Hipp (2019) it is shown that for $r p>1 / 2$ the function $\alpha \rightarrow V(s, \alpha)$ is continuous for all $s \geq 0$. The fact that the supremum is attained in (1) is obvious when $r p>1 / 2$. Without this condition, it is attained according to Lemma 2.e in Hipp (2003).

This Bellman equation is rather complex: the numerical computations need maximization over a continuous variable $0 \leq \beta_{1} \leq 1$, and the running ruin probability $\alpha(t)$ is defined in the optimization step. A reason for this complexity is the fact that our objective function present value of dividends is discounted while the constraint ruin probability is not. If we use the time value of ruin instead or a discounted penalty at ruin, then both functions are discounted with the same discount rate, and a normal Bellman equation can be used (see Albrecher and Thonhauser (2007) and Gerber et al. (2010)).

Here we introduce the class of two-barrier dividend strategies with the following property: when dividends are paid above a barrier $B$, i.e., a dividend of size 1 is paid when reaching $B+1$ from $B$, then we repeat this dividend payment at $B+1$ until reaching $L$ for some $0 \leq L \leq B$; after this, the next dividend payment happens at some $B_{1} \geq B$. For $L=B$ we pay a dividend of size 1 when reaching $B+1$ and then pay the next dividend when reaching $B_{1}+1$. For $L<B$ the sum of dividends paid at barrier $B+1$ as well as the time span in which we pay dividends at $B+1$ are random. For $L=B-1$ we continue paying dividends when reaching $B+1$ until a claim (a downward step) happens, and then we pay the next dividend when reaching $B_{1}+1$. When $L=B-2$, then we continue dividend payment at $B+1$ until we have two consecutive steps downward. If we keep paying dividends at $B+1$ until we have three consecutive steps down, then the resulting dividend strategy is not a two-barrier strategy.

Two-barrier strategies are defined by an initial value $S(0)=s_{0}$, by a sequence of barriers $s_{0} \leq B_{0} \leq B_{1}, \ldots$ above which we pay dividends, and by a sequence of levels $0 \leq L_{i} \leq B_{i}$ at which we stop paying dividends at $B_{i}+1$. The time period $\mathcal{P}_{i}$ in which we pay dividends at $B_{i}+1$ starts when - after starting in $s_{0}$ or after visiting $L_{i-1}$-we come to state $B_{i}+1$ where we pay a dividend of size 1 , and it ends when we enter the state $L_{i}$. We allow for $B_{i}=B_{i+1}$ but obtain different periods 
$\mathcal{P}_{i}$ and $\mathcal{P}_{i+1}$. When $B_{0}=2, B_{1}=2, B_{2}=3$ and $L_{0}=2, L_{1}=1, L_{2}=3$, and if our surplus process $S(t), t=0, \ldots, 10$, has the values $1,2,3,4,5,6,5,4,5,6,7$, then dividends of size 1 are paid at times $t=2,3,4,5,10$. After dividend payment, our surplus process evolves as 1,2, 2, 2, 2, 2, 1, 0, 1, 2, 2 . The periods are $\mathcal{P}_{0}=\{2\}, \mathcal{P}_{1}=\{3,4,5,6\}$, and $\mathcal{P}_{2}=\{10\}$.

We may restrict ourselves on sequences of barriers $B_{i}$ with $B_{i} \leq B_{i+1}$ : assume that for some $k \geq 0$ and $0<s<B$ the dividend strategy $\delta_{1}$ pays $k+1$ at $s+1$ after visiting $B+1$. Consider the dividend strategy $\delta_{2}$ paying $k$ at $s+1$ instead, and an additional 1 at $B+1$. This dividend strategy has the same ruin probability as $\delta_{1}$, but the present value of dividends of $\delta_{2}$ is larger than the one of $\delta_{1}$ since the payment of size 1 is paid earlier.

Notice that we first consider $s_{0} \leq B_{0}$; the case $s_{0}>B_{0}$ is covered in Section 3 .

The description of dividend strategies via the sequences $B_{i}, L_{i}$ is not handsome. An alternative method using barrier limits is presented in Section 7.

\section{Present Values of Dividends}

We denote by $f(s)$ the infinite time survival probability with initial surplus $s \geq 0$,

$$
f(s)=1-\psi_{0}(s)=1-(q / p)^{s+1}, s \geq-1 \text {. }
$$

This function solves

$$
f(s)=p f(s+1)+q f(s-1), s \geq 0,
$$

and it is the unique solution of this equation with $f(-1)=0, f(\infty)=1$. For integers $0 \leq s \leq b$ the probability $p(s, b)$ for reaching $b$ from $s$ before ruin equals $f(s) / f(b)$. This follows from the fact that a solution of (6) is uniquely defined by two values at $s_{1}$, $s_{2}$ with $-1 \leq s_{1}<s_{2}$. Clearly, $p(-1, b)=0$ and $p(b, b)=1$. For $0 \leq b<s$ the probability is different:

$$
p(s, b)=(q / p)^{b-s}
$$

Next we consider the score function $w(s)$ which satisfies $w(-1)=0, w(0)=1$, and

$$
w(s)=r p w(s+1)+r q w(s-1), s \geq 0 .
$$

The function $w(s)$ can be written as

$$
w(s)=C_{1} z_{1}^{s}+C_{2} z_{2}^{s}
$$

where $0<z_{1}<1<z_{2}$ are the two solutions of $z=r p z^{2}+r q$, and $C_{1}, C_{2}$ are chosen such that $w(-1)=0$ and $w(1)=1$. For $0 \leq s \leq b$ the present value $p v(s, b)$ of a single payment of 1 when-starting from $s$-reaching $b$ before ruin equals $w(s) / w(b)$. For $s>b$ we have

$$
p v(s, b)=z_{1}^{s-b}
$$

Furthermore, for $b \leq K$ the maximal present value of dividends paid above barrier $b \leq K$ when starting at $b$ equals $w(b) /(w(b+1)-w(b))$.

A third function is needed here: $v(s), s \geq-1$, which is defined as $v(s)=v_{1}(s)$, where $v_{1}(k)$ is the solution to the equations

$$
\begin{aligned}
v_{1}(-1) & =1 \\
v_{1}(k) & =r p v_{1}(k+1)+r q v_{1}(k-1), 0 \leq k \leq s, \\
v_{1}(s) & =v_{1}(s-1) .
\end{aligned}
$$


The function $v(s)$ is not a solution for (7). Still it is useful in the context of dividend payment: for fixed barrier $b \geq 0$ the expected discounted time to ruin for the risk process starting at $b \leq K$ with dividend payment of 1 whenever we visit $b$, equals $v(b)$.

The general solution of $(9)$ is $v_{1}(k)=w(k+1)+a w(k)$, where

$$
a=\frac{w(s+1)-w(s)}{w(s)-w(s-1)}
$$

leads to (10). So,

$$
v(s)=\frac{w(s)^{2}-w(s+1) w(s-1)}{w(s)-w(s-1)} .
$$

The numerator of this expression for $v(s)$ can be simplified:

Lemma 1. For $s \geq 0$

$$
w(s)^{2}-w(s-1) w(s+1)=(q / p)^{s} .
$$

Proof. For $s=0$ this is a consequence of the initial values $w(-1)=0$ and $w(0)=1$. Assume that (12) holds for $s$. For $s+1$ we must consider

$$
A=w(s+1)^{2}-w(s) w(s+2) .
$$

Write one of the terms $w(s+1)$ and $w(s+2)$ using (7), i.e.,

$$
A=w(s+1)(w(s)-r q w(s-1)) /(r p)-w(s)(w(s+1)-r q w(s)) /(r p) .
$$

The term $w(s) w(s+1)$ cancels, and we obtain $A=r p\left(w(s)^{2}-w(s+1) w(s-1)\right) /(r q)$.

Similar representations are possible for arbitrary functions satisfying (7); e.g.,

$$
f(s)^{2}-f(s-1) f(s+1)=f(0)^{2}(q / p)^{s} .
$$

From Equation (7) which concerns the values of $w$ at points $s-1, s, s+1$ we easily derive a relation for points $s-m, s-k, s+1$ when $0 \leq k<m \leq s$ which is used later for a simplified formula for the present value of dividends.

Lemma 2. For integers $0 \leq k<m \leq s$ we have

$$
w(s-k)=\left(\frac{q}{p}\right)^{k} A(k, m) w(s+1)+\left(\frac{q}{p}\right)^{m-k} \frac{w(k)}{w(m)} w(s-m) .
$$

where

$$
A(k, m)=(w(m) w(k-1)-w(k) w(m-1)) / w(m) .
$$

Proof. We first deal with the case $m=k+1$ :

$$
w(s-k)=\frac{1}{w(k+1)} w(s+1)+\frac{q}{p} \frac{w(k)}{w(k+1)} w(s-k-1),
$$

(see (12)) and use induction on $k$. For $k=0$ (14) is equal to Equation (7) since $w(1)=1 /(r p)$. Assume that Equation (14) holds for some $0 \leq k$ and all $s \geq k$. Using (7) for $s-k-1$ instead of $s$ we get

$$
w(s-k-1)=r p w(s-k)+r q w(s-k-2) .
$$

Replacing $w(s-k)$ in this equation by the right-hand side of (14) we obtain 


$$
w(s-k-1)=\operatorname{rp}\left(\frac{w(s+1)}{w(k+1)}+\frac{q}{p} \frac{w(k)}{w(k+1)} w(s-k-1)\right)+r q w(s-k-2),
$$

or

$$
w(s-k-1)\left(1-r \frac{r q w(k)}{w(k+1)}\right)=r p \frac{w(s+1)}{w(k+1)}+r q w(s-k-2) .
$$

Since from (7)

$$
1-r \frac{q w(k)}{w(k+1)}=r p \frac{w(k+2)}{w(k+1)}
$$

we arrive at Equation (14) for $k+1$.

The proof for general $m>k$ will use induction on $m$. We first notice that

$$
\left(\frac{q}{p}\right)^{k}(A(k, m+1)-A(k, m))=\left(\frac{q}{p}\right)^{m-k} \frac{w(k)}{w(m) w(m+1)} .
$$

Assume that (13) holds for some $m \geq 1$ and all $k<m$ and $s \geq m$, and it holds for all $m$ and $k=m-1$. i.e., (14). In (13) we replace $w(s-m)$ by the right-hand side of Equation (14) with $m$ instead of $k$ and arrive at

$$
w(s-k)=\left(\frac{q}{p}\right)^{k} A(k, m) w(s+1)+\left(\frac{q}{p}\right)^{m+1-k} \frac{w(k)}{w(m+1)}+R
$$

where

$$
R=\left(\frac{q}{p}\right)^{m-k} \frac{w(k)}{w(m) w(m+1)} w(s+1) .
$$

With (15) this yields the right-hand side of (13) for $m+1$.

The survival probability $s p\left(s_{0}\right)$ of a dividend strategies with parameters $s_{0} \leq B_{0} \leq B_{1}, \ldots$ and lower limits $L_{i} \leq B_{i}$ is given by

$$
s p\left(s_{0}\right)=f\left(s_{0}\right) \prod_{i=0}^{\infty} \frac{f\left(L_{i}\right)}{f\left(B_{i}+1\right)} .
$$

The survival probability equals the probability that the with dividend process reaches all states $B_{i}+1$ at which a dividend of size 1 is paid. The first barrier $B_{0}+1$ is reached with probability $f\left(s_{0}\right) / f\left(B_{0}+1\right)$. From $B_{0}+1$ we come to $L_{0}$ with probability 1 . From there we reach $B_{1}+1$ with probability $f\left(L_{0}\right) / f\left(B_{1}+1\right)$, and repeating this we obtain the above equation with independence of increments.

The present value of dividend payments equals

$$
P V\left(s_{0}\right)=w\left(s_{0}\right) \sum_{i=0}^{\infty} \frac{D_{i}}{w\left(B_{i}+1\right)} \prod_{k=0}^{i-1} \frac{C_{k} w\left(L_{k}\right)}{w\left(B_{k}+1\right)}
$$

Here, $D_{i}$ is the present value of dividend payment in period $\mathcal{P}_{i}$, discounted to the time of first dividend payment at $B_{i}+1$, and $C_{i}$ are discount factors over the periods $\mathcal{P}_{i}$. These numbers can be expressed via the functions $w(s)$ and $v(s)$ : For this recall that in our setup we have $B_{i}-L_{i} \leq K$ : if $B_{i}-L_{i}>K$, then we would pay a dividend when reaching $B_{i}$ which is not true by definition of $B_{i}$.

Consequently, $D_{i}=w\left(T_{i}\right) /\left(w\left(T_{i}+1\right)-w\left(T_{i}\right)\right)$, where $T_{i}=B_{i}-L_{i} \leq K$. This is the present value of dividend payment until ruin, with constant barrier $T_{i}$, starting at $T_{i}$. Clearly, $D_{i}=1$ when $B_{i}=L_{i}$. Furthermore, $C_{i}=v\left(T_{i}\right)$ when $T_{i} \leq K$. Also here, $C_{i}=1$ when $T_{i}=0$. 
For illustration we compute the present value of dividends $P V_{0}$ and $P V_{1}$ for the first two periods $\mathcal{P}_{0}$ and $\mathcal{P}_{1}$. Starting at $0 \leq s_{0} \leq B_{0}$ we have to wait for the first dividend payment until we reach $B_{0}+1$. If $L_{0}=B_{0}$ then the total dividend paid at $B_{0}+1$ is $D_{0}=1$, and so in this case $P V_{0}=D_{0} w\left(s_{0}\right) / w\left(B_{0}+1\right)$. If $L_{0}<B_{0}$, then we replace the sum of all payments until we reach $L_{0}$ by one payment of $D_{0}$ at $B_{0}+1$. Also in this case we have $P V_{0}=D_{0} w\left(s_{0}\right) / w\left(B_{0}+1\right)$.

The dividend payment of the second period starts at $B_{1}+1$. Discounting over the time elapsed until reaching $B_{1}+1$ from $s_{0}$ is done by three factors: the factor $w\left(s_{0}\right) / w\left(B_{0}+1\right)$ for the time until reaching $B_{0}+1$, the factor $v\left(B_{0}-L_{0}\right)$ for the time of dividend payment in period $\mathcal{P}_{0}$, and the factor $w\left(L_{0}\right) / w\left(B_{1}+1\right)$ for the time of reaching $B_{1}+1$ from $L_{0}$. At $B_{1}+1$ we have a present value of dividend payments equal to $D_{1}$. Again with independence of increments we obtain the present value for the period $\mathcal{P}_{1}$

$$
\frac{w\left(s_{0}\right)}{w\left(B_{0}+1\right)} \frac{w\left(L_{0}\right)}{w\left(B_{1}+1\right)} C_{0} D_{1} .
$$

For convenience we write ratios which depend on one index only, so the term for $\mathcal{P}_{1}$ is

$$
w\left(s_{0}\right) \frac{D_{1}}{w\left(B_{1}+1\right)} \frac{C_{0} w\left(L_{0}\right)}{w\left(B_{0}+1\right)} .
$$

Simplifications are possible for the case that $T_{i}=T \geq 0$ does not depend on $i$. For a strategy starting dividend payment at $B_{0}+1 \geq s_{0} \geq 0$ and $b_{i}=b_{0}+i, i=0,1,2, \ldots$, let $Z(b)$ be the number of barriers $B_{j}$ satisfying $B_{j}=b . Z(b)=0$ is allowed to deal with barriers which do not occur. Then the survival probability equals

$$
s p\left(s_{0}\right)=f\left(s_{0}\right) \prod_{b=B_{0}}^{\infty}\left(\frac{f(b-T)}{f(b+1)}\right)^{Z(b)},
$$

and the present value of dividend payments equals

$$
P V\left(s_{0}\right)=P v+w\left(s_{0}\right) \sum_{b=B_{0}}^{\infty} \prod_{s=B_{0}}^{b} h(s)^{Z(s)} \alpha(b) .
$$

Here, $P v$ is the present value of dividends paid above a fixed barrier $B_{0}$, starting in $s_{0}$,

$$
P v=\frac{w\left(s_{0}\right)}{w\left(B_{0}+1\right)-w\left(B_{0}\right)},
$$

and the function $h(s)$ is given by

$$
h(s)=v(T) w(s-T) / w(s+1), s \geq T \geq 0,
$$

while

$$
\alpha(s)=\frac{1}{w(s+2)-w(s+1)}-\frac{1}{w(s+1)-w(s)} .
$$

For the proof of (19) we start from (17): with $D=D_{i}$ and $C=C_{i}$ we obtain 


$$
\begin{aligned}
P V\left(s_{0}\right) & =w\left(s_{0}\right) D \sum_{b=B_{0}}^{\infty} \prod_{s=B_{0}}^{b-1} h(s)^{Z(s)} \frac{1}{w(b+1)} \sum_{j=0}^{Z(b)-1} h(b)^{j} \\
& =w\left(s_{0}\right) D \sum_{b=B_{0}}^{\infty} \prod_{s=B_{0}}^{b-1} h(s)^{Z(s)} \frac{1}{w(b+1)} \frac{1-h(b)^{Z(b)}}{1-h(b)} \\
& =P v+w\left(s_{0}\right) D \sum_{b=B_{0}+1}^{\infty} \prod_{s=B_{0}}^{b-1} h(s)^{Z(s)} \frac{1}{w(b+1)} \frac{1}{1-h(b)} \\
& -w\left(s_{0}\right) D \sum_{b=B_{0}}^{\infty} \prod_{s=B_{0}}^{b} h(s)^{Z(s)} \frac{1}{w(b+1)} \frac{1}{1-h(b)} \\
& \left.=P v+w\left(s_{0}\right) D \sum_{b=B_{0}}^{\infty} \prod_{s=B_{0}}^{b} h(s)^{Z(s)} \alpha_{1}(b),\right)
\end{aligned}
$$

where

$$
\alpha_{1}(b)=\frac{1}{w(b+2)} \frac{1}{1-h(b+1)}-\frac{1}{w(b+1)} \frac{1}{1-h(b)} .
$$

Here we used that $w\left(s_{0}\right) D /\left(1-h\left(b_{0}\right)\right)$ corresponds to a dividend strategy with $Z\left(B_{0}\right)=\infty$, which has a present value of payments equal to $P v$. With the same argument we can see that $D \alpha_{1}(s)$ does not depend on $T$, so it is equal to $\alpha(s)$ which corresponds to $T=0$. This proves (19).

A similar result can be derived when we have a finite number of possible values for $T_{i}$. For this we first show that dividend strategies can be permuted at a fixed barrier $B$ without changing the corresponding ruin probability as well as the present value of dividend payments.

Lemma 3. Let $s_{0}$ be the initial value of our risk process. Consider a dividend strategy defined by a sequence of barriers $s_{0} \leq B_{0} \leq B_{1}, \ldots$ and by a sequence of limits $0 \leq L_{i} \leq B_{i}$ Assume that for some $j \geq 0$ we have $B_{j}=B_{j+1}=b$, and $0 \leq k<m \leq b$ such that $L_{j}=b-k$ and $L_{j+1}=b-m$. Construct a second dividend strategy by the barriers $B_{0}, \ldots, B_{j-1}, B_{j+1}, B_{j}, B_{j+2}, B_{j+3}, \ldots$ and by the corresponding limits. Then the two dividend strategies have the same survival probability and the same present value of dividend payments.

Proof. The assertion concerning the survival probabilities is obvious. Looking at our formula (17) we see that interchanging the two-barriers has no effect on the terms in the sum with index $i<j$ and $i>j+1$. The quantities which might be changed by reversing the order are in the two terms with index $j, j+1$, they read

$$
\frac{1}{w(b+1)} D_{j}+\frac{1}{w(b+1)} C_{j} \frac{w(b-k)}{w(b+1)} D_{j+1}
$$

and

$$
\frac{1}{w(b+1)} D_{j+1}+\frac{1}{w(b+1)} C_{j+1} \frac{w(b-m)}{w(b+1)} D_{j} .
$$

These two terms are equal whenever

$$
w(b-k)=\frac{d_{m}-d_{k}}{d_{m} c_{k}} w(b+1)+\frac{d_{k} c_{m}}{d_{m} c_{k}} w(b-m)
$$

where the notation $d_{k}=D_{j}, d_{m}=D_{j+1}, c_{k}=C_{j}, c_{m}=D_{j+1}$ is chosen to indicate that these quantities depend on $k$ and $m$ only. According to Lemma 2, Equation (23) holds whenever

$$
\frac{d_{m}-d_{k}}{d_{m} c_{k}}=\left(\frac{q}{p}\right)^{k} A(k, m)
$$


and

$$
\frac{d_{k} c_{m}}{d_{m} c_{k}}=\left(\frac{q}{p}\right)^{m-k} \frac{w(k)}{w(m)} .
$$

Recall that

$$
d_{k}=\frac{w(k)}{w(k)-w(k-1)}
$$

and

$$
c_{k}=v(k)=\left(\frac{q}{p}\right)^{k} \frac{1}{w(k)-w(k-1)} .
$$

(see (11) and (12)). So

$$
d_{k} / c_{k}=w(k)\left(\frac{p}{q}\right)^{k}
$$

which yields (25). Furthermore,

$$
\begin{aligned}
\frac{d_{m}-d_{k}}{d_{m} c_{k}} & =\frac{1}{c_{k}}-\frac{d_{k}}{c_{k}} \frac{1}{d_{m}} \\
& =\left(\frac{p}{q}\right)^{k}\left(w(k)-w(k-1)-\frac{w(k)}{w(m)}(w(m)-w(m-1))\right. \\
& =\left(\frac{p}{q}\right)^{k} A(k, m)
\end{aligned}
$$

proves (24).

We now state the main result of this section, the representation for present values of dividend payments with a finite number of possible values for $T_{i}=B_{i}-L_{i}$ :

Proposition 1. Assume that for initial state $s_{0}$ and a dividend strategy with first dividend payment at $B_{0}+1$ we have $m+1$ possible values $0,1, \ldots, m$ for $T_{i}=B_{i}-L_{i}$. Then the survival probability of the with dividend process equals

$$
s p\left(s_{0}\right)=f\left(s_{0}\right) \prod_{k=B_{0}}^{\infty} \prod_{j=0}^{m}(f(k-j) / f(k+1))^{Z_{j}(k)},
$$

and the present value of dividend payments is given by

$$
P V\left(s_{0}\right)=P v+w\left(s_{0}\right) \sum_{b=B_{0}}^{\infty} \prod_{k=B_{0}}^{b} \prod_{j=0}^{m} h_{j}(k)^{Z_{j}(k)} \alpha(k) .
$$

Here, $Z_{j}(k)$ is the number of barriers $B_{i}$ with $B_{i}=k$ and $T_{i}=j$, and for $j=0, \ldots, m$

$$
h_{j}(k)=v(j) w(k-j) / w(k+1)
$$

and $P v$ is defined in (20).

Proof. The formula for the survival probability is straightforward. For the present value of dividends we first consider all discounted dividends which are paid when reaching $b+1$ from state $b$ for some $b \geq B_{0}$. Discounting for the time $t$ until reaching $b+1$ for the first time is done by the factor 


$$
w\left(s_{0}\right) \frac{1}{w(b+1)} \prod_{k=B_{0}}^{b-1} \prod_{j=0}^{m} h_{j}(k)^{Z_{j}(k)} .
$$

The time $t$ present value of the dividend payments is

$$
P v(b)=\sum_{k=0}^{m} \prod_{j=0}^{k} h_{j}(b)^{Z_{j}(b)} d_{k} \frac{1-h_{k}(b)^{Z_{k}(b)}}{1-h_{k}(b)} .
$$

Recall that $d_{k}=w(k) /(w(k)-w(k-1))$ and that $d_{k} /\left(1-h_{k}(b)\right)$ does not depend on $k$. Therefore, most of the terms in the sum $\mathrm{Pv}(b)$ cancel and we arrive at

$$
P v(b)=\frac{1}{1-h_{0}(b)}\left(1-\prod_{j=0}^{m} h_{j}(b)^{Z_{j}(b)}\right) .
$$

For the formula for $P V$ we use that the first term in $P v\left(B_{0}\right)$ equals $P v$, and that the other terms are generated by adding the corresponding two terms in $P v(b)$ and $P v(b+1)$ for $b \geq B_{0}$.

We may (and will) assume throughout that $B_{0} \geq K$, since $\alpha(s)>0$ for $s<K$ implies that we can increase $P V$ by the choice $Z_{j}(b)=0, b<K, j=0, \ldots, m$ without decreasing the corresponding survival probability. Furthermore, in case $s_{0} \leq K$ the present value of dividends can be written with $B_{0}$ replaced by $K$, when we use $Z_{j}(b)=0$ for $b<B_{0}$. This leads to a new value for $P v$, but this is compensated by the terms in the sum with $b<B_{0}$.

The formulas for survival probability and present value of dividends allow us to restrict our search for optimal dividend strategies considerably. Assume that for some fixed barrier $B$ we pay dividends at $B+1$ until reaching limit $L=B-T$. For the survival probability this generates the factor

$$
s p_{0}=f(B-T) / f(B+1),
$$

and in the sum representing the present value we obtain the factor

$$
p v_{0}=v(T) w(B-T) / w(B+1) .
$$

If we replace this dividend payment by $m$ payments at $B+1$ with limit $B$ and one payments at $B+1$ with limit $B-T+1$, these factors are

$$
s p_{1}=\left(\frac{f(B)}{f(B+1)}\right)^{m}\left(\frac{f(B-T+1)}{f(B+1)}\right)
$$

and

$$
p v_{1}=\left(\frac{w(B)}{w(B+1)}\right)^{m}\left(v(T-1) \frac{w(B-T+1)}{w(B+1)}\right)
$$

respectively. This leads to larger survival probability whenever $s p_{0} \leq s p_{1}$, and to a larger present value of dividends when $p v_{0} \geq p v_{1}$ (notice that the factors $\alpha(b)$ are all negative). Rewriting these inequalities, we obtain the following

Proposition 2. Assume that for all $T \geq 2$ and $s \geq K$ we can find an integer $m \geq 1$ such that

$$
\begin{aligned}
& \left(\frac{f(s)}{f(s+1)}\right)^{m} \geq \frac{f(s-T)}{f(s-T+1)} \\
& \left(\frac{w(s)}{w(s+1)}\right)^{m} \leq \frac{w(s-T)}{w(s-T+1)} \frac{v(T)}{v(T-1)}
\end{aligned}
$$


then we can restrict our search for optimal two-barrier dividend strategies on the set of strategies with exponents satisfying $Z_{j}(b)=0$ for all possible barriers $b \geq K$ and all $j \geq 2$.

The disadvantage of this result is the verification of inequalities (28) and (29), in particular since the exponent $m$ will not only depend on the model parameters $r$ and $p$, but also on $T$. However, it often (but not always) can be chosen independent of $s$. Sometimes, only detailed inspection works. Recall that $w(s)=C\left(z_{1}^{s}-z_{2}^{s}\right), s \geq 0$, with $0<z_{1}<1<z_{2}, C_{1}<0<C_{2}=-C_{1} z_{2} / z_{1}$. We write $a$ for $q / p$, i.e., $f(s)=1-a^{s}$.

Lemma 4. For fixed $T \geq 2$ and some integer $m \geq 1$ let

$$
\begin{gathered}
F(s)=\left(\frac{f(s)}{f(s+1)}\right)^{m}-\frac{f(s-T)}{f(s-T+1)}, s \geq T, \\
G(s)=\left(\frac{w(s)}{w(s+1)}\right)^{m}-\frac{w(s-T)}{w(s-T+1)} \frac{v(T)}{v(T-1)}, s \geq T .
\end{gathered}
$$

Then

(a) $F(s) \geq 0$ for all $s \geq T$ is possible iff $m \leq a^{-T}$.

(b) $G(s) \leq 0$ for all $s \geq T$ is possible only if $z_{2}^{m-1} \geq v(T-1) / v(T)$. Under this condition, if for some $s_{1} \geq T$ we have

$$
\left(z_{2} \frac{w(s)}{w(s+1)}\right)^{m}-z_{2} \frac{w(s-T)}{w(s-T+1)} \leq 0,
$$

then $G(s) \leq 0$ for all $s \geq s_{1}$.

An integer m satisfying both conditions-without (30)—will exist whenever

$$
a^{-T}-\log (v(T-1) / v(T)) / \log \left(z_{2}\right) \geq 2 .
$$

Proof. For both assertions we look at the asymptotic behavior for $s \rightarrow \infty$.

(a) Let $g(s)=\log (f(s) / f(s+1))$. Then $F(s) \geq 0$ is equivalent to $m g(s) \geq g(s-T)$ or

$$
m g(s) / g(s-T) \leq 1
$$

(notice that $g(s-T)<0)$. Since $\log (f(s))=\log \left(1-a^{s}\right)=a^{s}(1-a)+o\left(a^{s}\right), s \rightarrow \infty$, we obtain

$$
\lim _{s \rightarrow \infty} \frac{m g(s)}{g(s-T)}=m a^{T}
$$

which does not exceed 1 if $m \leq a^{-T}$. Under this condition, we have $F(s) \geq 0$ for all $s \geq T$ since $g(s) / g(s-T)$ is increasing. To show this it is enough to consider $T=1$ (the product of positive increasing functions is increasing). We have

$$
\begin{aligned}
g^{\prime}(s) & =-\log (a)(1-a) \frac{a^{s}}{f(s) f(s+1)}>0, \\
g^{\prime \prime}(s) & =-\log (a)^{2}(1-a) \frac{\left.a^{s} f(2 s+1)\right)}{f(s)^{2} f(s+1)^{2}}<0 . \\
g^{\prime}(s+1) & =a g^{\prime}(s) f(s) / f(s+2) .
\end{aligned}
$$

Here we consider $g$ as a function of a real variable. Notice first that the function

$$
u(s)=a f(s) g(s) / f(s+1)-g(s+1), s>0,
$$


is decreasing, since

$$
\begin{aligned}
u^{\prime}(s) & =a\left(\frac{f(s)}{f(s+1)}\right)^{\prime} g(s)+a\left(\frac{f(s)}{f(s+1)}\right) g^{\prime}(s)-g^{\prime}(s+1) \\
& =a\left(\frac{f(s)}{f(s+1)}\right)^{\prime} g(s)<0 .
\end{aligned}
$$

Since $u(s) \rightarrow 0$ for $s \rightarrow \infty$ we have $u(s)>0$ for $s>0$. Now, we can show that $g(s+1) / g(s)$ is increasing: with $g(s) / f(s+2) \geq g(s) / f(s+1)$

$$
\begin{aligned}
\left(\frac{g(s+1)}{g(s)}\right)^{\prime} & =\frac{g(s) g^{\prime}(s+1)-g^{\prime}(s) g(s+1)}{g(s)^{2}} \\
& =\frac{g(s) g^{\prime}(s) a f(s) / f(s+2)-g^{\prime}(s) g(s+1)}{g(s)^{2}} \\
& \geq \frac{g(s) g^{\prime}(s) a f(s) / f(s+1)-g^{\prime}(s) g(s+1)}{g(s)^{2}} \\
& =g^{\prime}(s) u(s) / g(s)^{2} \geq 0 .
\end{aligned}
$$

(b) Let $h(s)=w(s) / w(s+1)$. Then $G(s+T) \leq 0$ is equivalent to

$$
h(s+T)^{m} \leq h(s) v(T) / v(T-1) .
$$

Using the relation

$$
w(s)=\frac{z_{1}^{s}-z_{2}^{s}}{z_{1}-z_{2}}
$$

we obtain

$$
h(s)=\frac{1}{z_{2}} \frac{1-a_{0}^{s}}{1-a_{0}^{s+1}}
$$

with $0<a_{0}=z_{1} / z_{2}<1$. We have $h(s) \rightarrow 1 / z_{2}$ and so the necessary condition is $z_{2}^{m-1} \geq v(T-1) / v(T)$. If this holds, then a sufficient condition for $G(s+T) \leq 0$ is

$$
\left(z_{2} h(s+T)\right)^{m} \leq z_{2} h(s) .
$$

If (31) is true for $s_{1}$, then

$$
\log \left(z_{2} h\left(s_{1}+T\right)\right) / \log \left(z_{2} h\left(s_{1}\right)\right) \geq 0 .
$$

The proof of part (a) with $a=q / p$ replaced by $a=z_{1} / z_{2}$ and $z_{2} h(s)$ instead of $f(s) / f(s+1)$ yields that

$$
\log \left(z_{2} h(s+T)\right) / \log \left(z_{2} h(s)\right)
$$

is increasing, and so (31) holds for all $s \geq s_{1}$.

In our numerical experiments, we have $K=4$, so we first checked the validity of conditions (28) and (29) for $T=3,4$ and then we searched for dividend strategies with maximal present value in the set of two-barrier strategies which have limits $L_{i}=B_{i}$ or $L_{i}=B_{i}-1$.

\section{Immediate Dividend Payment}

Here we consider the case that our initial surplus is larger than $B_{0}$. In this situation, we could decide to reduce the allowed ruin probability (which will increase $B_{0}$ ), or we can use the extra surplus 
$s_{0}-B_{0}$ to increase the company value, or we could do both. In this section we want to use the extra surplus to increase the company value.

Assume first that $s_{0}=B_{0}+1$. Let $\alpha$ be the allowed ruin probability for initial surplus $s_{0}$. We can pay a dividend of size 1 immediately, which leads to

$$
V\left(s_{0}, \alpha\right)=1+V\left(B_{0}, \alpha\right) .
$$

Or we can start a $T=1$-dividend payment which pays 1 each time we reach $B_{0}+2$, until the first downward step which leads us to $B_{0}$ :

$$
V\left(s_{0}, \alpha\right)=A(1)+C(1) V\left(B_{0}, \alpha\right) .
$$

If we maximize the dividend value, our action will be the immediate dividend payment of 1 when the value in (32)is larger than the one in (33). Otherwise, we choose the $T=1$-payment. We have $A(1)>1$ and $C(1)<1$, so both cases could happen. The condition for the $T=1$-payment is

$$
V(B(s), \alpha) \leq(A(1)-1) /(1-C(1)) .
$$

We see that using the extra surplus $s_{0}-B_{0}$, we can maximize the present value of total dividends by the choice of an appropriate dividend strategy. Notice that the allowed ruin probability is achieved automatically after hitting $B_{0}$. Therefore, the optimization of a dividend strategies is easier, it can be done without considering ruin probabilities. We use a recursive optimization: assume that an optimal strategy is available for initial surplus $s_{1}>B_{0}$ with $V\left(s_{1}, \alpha\right)$ as present value of dividend payments.

Lemma 5. Assume that

$$
V\left(B_{0}, \alpha\right)>(A(2)-A(1)-C(1) A(1)) /(C(1)-C(2) .
$$

Then the optimal strategy at surplus $s_{0}=s_{1}+1$ is either an immediate payment of 1 producing $V\left(s_{0}, \alpha\right)=1+V\left(s_{1}, \alpha\right)$, or a $T=1$-payment at $s_{0}$ (pay a dividend of size 1 when reaching $s_{1}+1$ until you hit $\left.s_{1}\right)$, which yields $V\left(s_{0}, \alpha\right)=A_{1}+C_{1} V\left(s_{1}, \alpha\right)$. The optimal choice will be $T=1$ iff

$$
V\left(s_{1}, \alpha\right) \leq(A(1)-1) /(1-C(1)) .
$$

Proof. Consider first a $T=2$-payment starting at $s_{0} \geq B(s)+2$, i.e., repeated payment of 1 at state $s_{0}+1$ until the first step downward which leads us to $s_{0}-2 \geq B(s)$. The present value of dividends equals

$$
V_{1}=A(2)+C(2) V\left(s_{0}-2, \alpha\right) .
$$

As an alternative, consider two concatenated $T=1$-payments, the first starting at $s_{0}+1$ and ending when hitting $s_{0}-1$, the second starting at $s_{0}$ and ending at $s_{0}-2$. The present value of this strategy is

$$
V_{2}=A(1)+C(1)\left(A(1)+C(1) V\left(s_{0}-2, \alpha\right)\right) .
$$

The difference is

$$
V_{2}-V_{1}=A(2)-A(1)-C(1) A(1)+(C(1)-C(2)) V\left(s_{0}-2, \alpha\right)
$$

which is positive because of (35) and $V\left(s_{0}-2, \alpha\right) \geq V\left(B_{0}, \alpha\right)$. Therefore, the $T=1$-payment is better at $s_{0} \geq B_{0}+2$. If $s_{0}=B_{0}+1$ then the present values $V_{1}, V_{2}$ are the same, up to a factor $w\left(s_{0}\right) / w\left(s_{0}+1\right)$ which represents discounting to reach $s_{0}+1$ from $s_{0}$ without dividend payment. 
If a $T$-payment would be optimal at $s_{0}$ for some $T \geq 3$, then a $T-1$-payment would be optimal at $s_{0}-1$, so under condition (35) a $T=1$-payment is better than any $T$-payment for $T \geq 2$. This proves the lemma.

Notice that (35) is a weak condition in the sense that it is not easy to find cases in which it is not satisfied; for this, $V(B(s), \alpha)$ must be close to zero, and $A(2)-A(1)-A(1) C(1)<0$ is always true.

The resulting dividend strategy is a $T=1$-payment for all $s \geq B(s)$ up to the boundary given in (34), above it is an immediate lump sum of $s-B(s)$. A numerical example is given in Section 7.

\section{Running Survival Probabilities}

Assume that an initial value $s_{0}$, and a sequence of barriers $s_{0} \leq B_{0} \leq B_{1} \ldots$ and limits $0 \leq L_{i} \leq B_{i}$ are given. For $t \geq 0$ we here compute the running survival probabilities $s p(t)$ and the present values of dividends $P V(t)$ for time $t$ and for the dividend strategy $\delta(t)$ defined by the above quantities. Clearly, $s p(t)$ will not only depend on $S^{\delta}(t)$, the risk process with dividend payment. We will see that for $t \geq 1 s p(t)$ is a function of $X_{t}, S^{\delta}(t-1)$, and $s p(t-1)$.

At time $t=0$ we have-according to (16) -with our initial surplus $s_{0}$ a with dividend survival probability

$$
1-\alpha=f\left(s_{0}\right) \prod_{i=0}^{\infty} f\left(L_{i}\right) / f\left(B_{i}+1\right)
$$

As long as no dividends are paid, the survival probability at state $s \leq B_{0}$ is given by

$$
f_{0}(s)=f(s) \prod_{i=0}^{\infty} f\left(L_{i}\right) / f\left(B_{i}+1\right)=f(s)(1-\alpha) / f\left(s_{0}\right),
$$

which follows from (16) when starting at $s$ instead of $s_{0}$.

After reaching $B_{0}+1$, dividend payment leads us to the state $L_{0}$ with probability 1 . After hitting $L_{0}$, we will not pay dividends until we reach the next barrier $B_{1}+1$. Formula (16) with initial surplus $L_{0}$ and dividend payment after reaching $B_{1}$ gives us the survival probability

$$
f\left(L_{0}\right) \prod_{i=1}^{\infty} f\left(L_{i}\right) / f\left(B_{i}+1\right)
$$

which is the same as

$$
f\left(B_{0}+1\right) \prod_{i=0}^{\infty} f\left(L_{i}\right) / f\left(B_{i}+1\right)=f_{0}\left(B_{0}+1\right) .
$$

We obtain that after starting dividend payment at $B_{0}+1$, the survival probabilities at states $s=L_{0}, L_{0}+1, \ldots, B_{0}+1$ are all equal to the value in (38).

Now we repeat this argument for each barrier $B_{i}, i \geq 1$ : for states $s$ reached at or after visiting $L_{i-1}$ and before hitting the state $B_{i}+1$ the survival probability equals

$$
f_{i}(s)=f(i) \prod_{m=i}^{\infty} f\left(L_{m}\right) / f\left(B_{m}+1\right)
$$

while for all states $s=L_{i}, L_{i}+1, \ldots, B_{i}+1$ which are reached after the first dividend payment at $B_{i}+1$ we have the same survival probability $f_{i}\left(B_{i}+1\right)$. These functions are defined for $s=-1, \ldots, B_{i}+1$ and concatenated by the equation

$$
f_{i+1}\left(L_{i}\right)=f_{i}\left(B_{i}+1\right), i \geq 0,
$$

(see the two equations for $i=0$ above). 
We now show that for $t \geq 1$ the survival probability $s p(t)$ is determined by the values $S^{\delta}(t-1)$, $s p(t-1)$, and $X_{t}$. From the values $s p(t-1)$ and $s=S^{\delta}(t-1)$ we can see for which index $i$ the survival probability $s p(t-1)$ can be expressed by $f_{i}(s)$ when $s=S^{\delta}(t-1)$. This covers the two cases

(a) at time $t-1$ we have not yet visited $B_{i}+1$, but have visited $L_{i-1}$ before, and

(b) we have paid a dividend at $B_{i}+1$ before time $t-1$, and $L_{i}<s \leq B_{i}+1$.

For short, let $a_{i}=f_{i}\left(B_{i}+1\right) / f\left(B_{i}+1\right)$.

In case (a) we have $s p(t-1)=a_{i} f(s)$ for $s=-1,0,1, \ldots, B_{i}$, while in case (b) we get $s p(t-1)=f_{i}\left(B_{i}+1\right)$ for $s=L_{i}+1, \ldots, B_{i}+1$. So $s p(t-1)$ and $s$ tell us in which of the two cases we are.

If $X_{t}=1$, then in case (a), $s p(t)=a_{i} f(s+1)$, and in case (b) we obtain $s p(t)=s p(t-1)$. If $X_{t}=-1$ and $s=S^{\delta}(t-1) \geq 1$, then $s p(t)=a_{i} f(s-1)$, and in case (b) we have $\operatorname{sp}(t)=\operatorname{sp}(t-1)$ when $s>L_{i}+1$. For $s=L_{i}+1$ we obtain $s p(t)=s p(t-1)$ from (39).

Remark 1. When the search for optimal dividend strategies is restricted to the set of two-barrier strategies, in the Bellman Equation (1) the supremum is just over the two pairs

$$
\begin{aligned}
& \beta_{1}=\alpha f(s+1) / f(s), \beta_{2}=\alpha f(s-1) / f(s), \\
& \beta_{1}=\beta_{2}=\alpha .
\end{aligned}
$$

\section{Lagrange Approach and Derivatives}

In Equations (18) and (19) we now replace the numbers $Z(b)$ of iterations by real numbers $R(b)$ and use infinitesimal calculus to find optimal exponents, using the method of a Lagrange multiplier. Our initial values satisfy $B_{0} \geq K$ and $s_{0} \leq B_{0}$. For $L>0$ we consider

$$
P V\left(s_{0}\right)+L \log \left(s p\left(s_{0}\right)\right)
$$

and the corresponding normal equations. The system of partial equations for $R(b), b \geq B_{0}+1$, can be solved explicitly, and the solution does not depend on $L$ :

$$
\begin{aligned}
\beta(b) & =\log (f(b-T) / f(b+1)) / \log (h(b)) \\
P(b) & =L \frac{\beta(b)-\beta(b+1)}{\alpha(b)} \\
R(b) & =\frac{\log (P(b))-\log (P(b-1))}{\log (h(b))} .
\end{aligned}
$$

For $b=B_{0} R(b)$ is the solution of

$$
w\left(s_{0}\right) h(b)^{R(b)}=P(b)
$$

Since $L$ appears only in the definition of $R\left(B_{0}\right)$, we can define $R\left(B_{0}\right)$ from the given allowed ruin probability $\alpha$ and all the quantities $R(b), b \geq B_{0}+1$ as the solution of the equation

$$
\left(\frac{f\left(B_{0}-T\right)}{f\left(B_{0}+1\right)}\right)^{e x} f\left(s_{0}\right) \prod_{s=B_{0}+1}^{\infty}\left(\frac{f(s-T)}{f(s+1)}\right)^{R(s)}=1-\alpha .
$$

Then

$$
V_{R}=P v+w\left(s_{0}\right) \sum_{b=B_{0}}^{\infty} \prod_{s=B_{0}}^{b-1} h(s)^{R(s)} \alpha(b) .
$$


provides an upper bound for the present value of dividend payments $V(s, \alpha)$ with survival probability $1-\alpha$. In our numerical experiments below we use the floors of $R(b), b \geq B_{0}$, as an initial sequence of exponents for an improvement process.

For the case with $m+1$ possible values $0,1, \ldots, m$ for $T_{i}=B_{i}-L_{i}$, for each $b \geq 0$ we have $m+1$ exponents $R_{j}(b), j=0, \ldots, m$. In this situation the normal equations for the Lagrange multiplier approach (42) imply that for each $b \geq K$ there is at most one index $j$ with $R_{j}(b)>0$, and this index maximizes

$$
H_{k}(b)=\frac{\log (f(b-k) / f(b+1))}{\log \left(h_{k}(b)\right)}, k=0, \ldots, m .
$$

This might indicate that also in the discrete case, with integer exponents $Z_{j}(b)$ instead of real values $R_{j}(b)$, one will have only one index $j$ satisfying $Z_{j}(b)>0$. However, in our numerical experiments the maximal present values of dividends were never achieved with only one exponent $Z_{j}(b)>0$ for all $b$. Examples and computations for mixed strategies are given below.

For the standard parameters $p=0.7$ and $r=1 / 1.03$ we find that for all $b \geq K$ we have the minimum for (46) at $k=1$, as you can read from the following Table 1.

Table 1. Critical ratios in (46).

\begin{tabular}{cccccccc}
\hline $\boldsymbol{b}$ & $\boldsymbol{H}_{\mathbf{0}}(\boldsymbol{b})$ & $\boldsymbol{H}_{\mathbf{1}}(\boldsymbol{b})$ & $\boldsymbol{H}_{\mathbf{2}}(\boldsymbol{b})$ & $\boldsymbol{H}_{\mathbf{3}}(\boldsymbol{b})$ & $\boldsymbol{H}_{\mathbf{4}}(\boldsymbol{b})$ & $\boldsymbol{H}_{\mathbf{5}}(\boldsymbol{b})$ & $\boldsymbol{H}_{\mathbf{6}}(\boldsymbol{b})$ \\
\hline 6 & 0.02184 & 0.02154 & 0.0231 & 0.0284 & 0.0409 & 0.0680 & 0.1320 \\
\hline 7 & 0.00939 & 0.00927 & 0.0099 & 0.0121 & 0.0174 & 0.0284 & 0.0517 \\
\hline 8 & 0.00403 & 0.00397 & 0.0042 & 0.0052 & 0.0074 & 0.0120 & 0.0214 \\
\hline 9 & 0.00172 & 0.00170 & 0.0018 & 0.0022 & 0.0031 & 0.0051 & 0.0090 \\
\hline 10 & 0.00075 & 0.00073 & 0.00078 & 0.0009 & 0.0013 & 0.0021 & 0.0038 \\
\hline
\end{tabular}

\section{Discussion}

The computation of ruin probabilities and present value of dividends for two-barrier strategies in De Finetti models is rather simple, and at the same time these strategies are flexible enough to yield large dividend values (at least larger than those in earlier publications). Their computation can be done for a single initial value $s_{0}$ and allowed ruin probability $\alpha$. In addition, they can be computed using a simplified iteration method (see (5) and Remark (1)). The original iteration method (5) yields a globally optimal dividend value, but it is too demanding for numerical computations. It is unknown whether the simplified iteration method also produces a globally optimal dividend value. At least, it produces an optimal dividend value in the class of all two-barrier strategies. In our paper, we compute dividend strategies with large present value using explicit formulas for this value, derived for two-barrier strategies. There, one can use conditions (28) and (29) to restrict the set of possible strategies. However, the restriction on mixtures of $T=0$ - and $T=1$-strategies is possible only for normal values of $p$ and $\alpha$ as in our numerical examples. In more extreme situations (e.g., $p=0.55$ or $r=1 / 1.01$ ) Lemma 4 applies not for $T \geq 2$, but for $T \geq 4$ (or even larger) only. Recall that we have excluded $T>K$ before.

A general setup for optimal dividend payment with ruin constraint could be the following: for barriers $b=K, K+1, \ldots$ we define stopping times $\tau(b)$ which define the time span $S(b)$ in which dividends of size 1 are paid at $b$ :

$$
S(b)=\tau(K)+\ldots+\tau(b) \leq t<\tau(K)+\ldots+\tau(b+1) .
$$

For two-barrier strategies these stopping times have a special structure: if $Z_{j}(b), j=0, \ldots, m$ are the exponents for barrier $b$ and limit $b-j$, then $\tau(b)$ is the time needed to hit limit $b-j Z_{j}(b)$ times from $b+1$, for $j=0, \ldots, m$. Not all stopping times are of this form: if we stop when, after visiting $b+1$, we have three steps downward in a row, we clearly have a stopping time. This cannot be represented by a two-barrier strategy since we will never stop on the way to ruin via a sequence 
$s, s-1, s-2, s-1, s-2, s-3, s-2, s-3, s-4, \ldots$ On the other hand, using the concept of stopping times (which turn out to be independent) one could come to solution of our problem in the class of all admissible strategies. In particular, our Lemma 3 might become obvious. Our approach using two-barrier strategies is more direct and closer to computer programming.

When two or more values for $T$ are considered, we could select the optimal $T$ from the equation

$$
V(b+1, \alpha)=\max \{A(T)+C(T) V(b-T, \alpha), 0 \leq T \leq b\}
$$

which holds for the optimal two-barrier strategy and for all barriers $b$ with dividend payment at $b+1$.

Furthermore, this equation yields a relation between $V\left(b-T_{1}, \alpha\right)$ and $V\left(b-T_{2}, \alpha\right)$ if $b$ is a barrier for which dividends of two different types $T_{1}, T_{2}$ are paid; e.g., if $T=0$ - and $T=1$-payments are made at $b+1$ then

$$
\begin{aligned}
V(b+1, \alpha) & =1+V(b, \alpha), \\
V(b+1, \alpha) & =A(1)+C(1) V(b-1, \alpha), \text { or } \\
V(b, \alpha) & =A(1)-1+C(1) V(b-1, \alpha) .
\end{aligned}
$$

The numerical procedures used in our experiments are in line with these equations. For the standard model parameters and $\alpha=0.022602487$ we have $b=6, Z_{0}(6)=Z_{1}(6)=1$, and $V(6, \alpha)=12.78113592, V(7, \alpha)=1+V(6, \alpha)$, and $V(5, \alpha)=11.72591617879$ which yields (50).

There are more such intuitive relations between present values of dividends, and all of them are consistent with our numerical findings. If, e.g., $(Z(b), E(b)): b \geq 4$, is the sequence of exponents for the present value $V\left(s_{0}, \alpha\right)$ with $E\left(b_{0}\right)=1$, and dividend payment starting at $b_{0}+1$, then for

$$
\alpha_{1}=1-(1-\alpha) f\left(b_{0}+1\right) / f(b 0),
$$

the first sequence of exponents, with $E\left(b_{0}\right)$ replaced by 0 , is the sequence of exponents for $V\left(s_{0}, \alpha_{1}\right)$, and

$$
V\left(s_{0}, \alpha_{1}\right)=P v-w\left(b_{0}+1\right) / w\left(b_{0}\right)\left(P v-V\left(s_{0}, \alpha\right)\right) .
$$

Here, $P v=w\left(s_{0}\right) /\left(w\left(b_{0}+1\right)-w\left(b_{0}\right)\right.$. Changing the exponent $E\left(b_{0}\right)$ from 1 to 0 means that we omit a dividend payment of 1 at $b_{0}+1$, and $w\left(s_{0}\right) / w\left(b_{0}+1\right)$ is the discount factor for this payment. For a $T=1$-dividend payment at $b_{0}$ we obtain $\alpha_{1}=1-(1-\alpha) f\left(b_{0}+1\right) / f\left(b_{0}-1\right)$, and

$$
V\left(s_{0}, \alpha_{1}\right)=P v-w\left(b_{0}+1\right) /\left(v(1) w\left(b_{0}-1\right)\right)\left(P v-V\left(s_{0}, \alpha\right)\right) .
$$

Similar equations hold for the case when more than one dividend payments are omitted. These simple relations hold, however, only for the barrier $b_{0}$ at which dividends are paid first.

Two-barrier strategies should also be considered in other risk models such as Lundberg models or diffusion models. The computation of their survival probabilities as well as present value of dividends is as simple as in De Finetti models. In addition, they might perform better than the dividend strategies considered so far.

\section{Numerical Experiments}

We continue the numerical example in Hipp (2018) where $p=0.7, r=1 / 1.03, s_{0}=4$ and $\alpha=0.2$. As a result of many iterations given in (5), a value $V(4,0.2)=12.817618$ was obtained. The second value $V(4,0.2)=12.9099$ given in Hipp (2018) is wrong since the underlying dividend strategy has a ruin probability larger than 0.2 .

For the computation of a dividend strategy with given ruin probability $\alpha$ and $T=B_{i}-L_{i}$ independent of $i$, we start with the choice of a barrier $B_{0}$ at which dividends are paid first. Let $R(i)$ 
be the solutions of the normal equations for the Lagrange approach (42) and $B_{0}=b \geq K$ the smallest integer for which the solution $e x$ to the equation

$$
f_{1}(b)^{e x} \prod_{s=b+1}^{\infty} f_{1}(s)^{R(s)}=1-\alpha
$$

is positive, where $f_{1}(s)=f(s-T) / f(s+1), s \geq 0$.. As initial sequence of exponents we take $Z_{i}^{0}=$ floor $(R(i)), i>b$, and $Z_{b}^{0}=$ floor $(e x)$.

For $T=0$ we have $B_{0}=K=4$, and the exponents $Z_{i}^{0}$ are $Z_{4}^{0}=13, Z_{5}^{0}=18, Z_{6}^{0}=13$, $Z_{i}^{0}=11, i \geq B_{0}$, they define a strategy with ruin probability

$$
1-\prod_{s=4}^{\infty}\left(f_{1}(s)\right)^{Z_{s}^{0}}=0.196998263935
$$

We now increase these exponents sequentially and stepwise as long as the resulting ruin probability is smaller than $\alpha$. This produces a sequence of exponents $Z_{i}$ which, for $4 \leq i \leq 20$ read

$$
13,19,13,11,11,12,12,11,12,13,11,11,12,12,11,11,13
$$

The resulting ruin probability is almost 0.2 , and the dividend value is $V_{0}(4,0.2)=12.817726$. The same procedure is repeated with $Z_{i}^{1}=$ floor $(R(i))-1, i>B_{0}$. This sequence $Z_{i}^{1}$ produces a dividend strategy with the same ruin probability but with somewhat earlier payments. In the given situation we obtain a dividend value $\hat{V}_{0}(4,0.2)=12.81675$, and the initial terms for the sequence of exponents are

$$
14,17,13,1,10,10,11,11,12,10,10,10,11,11,12,10,10,10,11,10,11,10 .
$$

The second present value is not always smaller than the first, so we take the maximum of both as $V_{0}(s, \alpha)$.

For $T=1$ we obtain the same $K$ and $Z_{4}^{0}=3, Z_{5}^{0}=5, Z_{i}^{0}=3, i \geq 6$, and the sequence of exponents $Z_{i}$ for $4 \leq i \leq 20$ is

$$
4,5,4,5,3,4,4,3,4,3,3,3,5,3,4,4,4 \text {. }
$$

The dividend value for this setup is $V_{1}(4)=12.8225955$. We see that here, a pure $T=1-$ strategy is slightly better than the corresponding pure $T=0-$ strategy.

With starting value $s_{0}=1$ and $\alpha=0.2$ we obtain $b=6$. The pure $T=0-$ strategy has $V_{0}\left(s_{0}, \alpha\right)=7.79110112$, the $T=1-$ strategy yields $V_{1}\left(s_{0}, \alpha\right)=7.79412286$. Higher dividend values can be obtained with mixed strategies, i.e., mixing $T=0$-payments with $T=1$ - payments. In our experiments we used the above construction of $T=1$ - strategies for a given sequence of exponents for $T=0$ - payments. If a sequence of exponents $Z_{0}(i)$ for $T=0$ - payments is given, then we adjust the allowed ruin probability $\alpha_{0}$ accordingly:

$$
\alpha_{1}=1-\left(1-\alpha_{0}\right) \prod_{s=b}^{\infty}(f(s) / f(s+1))^{Z_{0}(i)}
$$

which is the allowed ruin probability for $T=1$-payments; here, $b$ is the barrier for the first dividend payment. For $\alpha_{1}$ we construct a pure $T=1$ - dividend strategy. The exponents $Z_{0}(i)$ are chosen sequentially according to the resulting dividend value of the combined strategy. For this problem we include a MatLab code in which the details can be seen. For the multiple loops in the program, MAPLE is not handsome. On the other hand, the limited numerical accuracy of MatLab allows the computation of a limited number of values for $R(s)$ only. This problem is solved via approximations. 
For $s_{0}=1$ and $\alpha=0.2$ we obtain $b=6$, and the following nonzero values $Z_{0}(i) \leq 3, i \leq 30$, are

$$
Z_{0}(i)=1, i=6,11,12,19,25,31,32,36 ; Z_{0}(i)=2, i=13,21, Z_{0}(44)=3,
$$

while the first 40 exponents $Z_{1}(i), i=6, \ldots, 45$, are all equal to 3 , except $Z_{1}(6)=1$ and $Z_{1}(i)=4$ for $i=7,16,28,34$. The dividend value equals $V(1, \alpha)=7.798392888749329$.

The restriction to two-barrier strategies with $T=0$ and $T=1$ only is justified by Proposition 2 and Lemma 4. For $T=2$, e.g., we have $v(T-1) / v(T)=1.3121 \ldots, z_{2}=1.07142857$ and $z_{2}^{4}=1.31781028738$, so $m=5$ is a possible exponent for which in addition $m a^{T}=0.918367<1$. In this case, however, condition (29) is not true for $s=4$, but this is not essential since we have a first barrier $b=6$ for which the inequality holds. The following Table 2 gives some results for strategies with only $T=0-$ and $T=1$ - payments, pure as well as mixed strategies. The columns labeled $T 0$ and $T 1$ show the dividend value for pure $T=0-$ and $T=1-$ strategies. The column $T 10$ shows the dividend value for a pure $T=1$-strategy which is mixed with single $T=0$ - payments chosen one by one according to the resulting dividend value. The column $T 01$ shows the corresponding values for a pure $T=0-$ strategy mixed with $T=1$ - payments. The initial surplus $s_{0}=1$ is the same for all cases. The smallest possible allowed ruin probability for $s_{0}=1$ is $f\left(s_{0}\right)=0.18367$.

We see that the T10-value is the largest in all cases except for $\alpha=0.2$ and $1 / r=1.07$. Furthermore, for $\alpha=0.185$ the pure T0-strategy is not improved by single $T=1$-payments and better than the pure $T=1$-strategy. This is not in line with formula (46): for $\alpha=0.2$ we have $b=6$, and $\alpha=0.185$ produces $b=9$. However, for both values of $b$ the maximum of (46) is at $k=0$ : for $b=6$, we have the values 0.02183599 for $k=0$ and 0.0215461488 for $k=1$, while for $b=9$ the values are 0.0017308 and 0.0017063 .

Table 2. Selection of numerical results.

\begin{tabular}{cccccc}
\hline $\boldsymbol{\alpha}$ & $\mathbf{1} / \boldsymbol{r}$ & $\mathbf{T 0}$ & $\mathbf{T 0 1}$ & $\mathbf{T 1}$ & $\mathbf{T 1 0}$ \\
\hline 0.2 & 1.02 & 12.71315904 & 12.71316594 & 12.71786387 & 12.71925617 \\
\hline 0.2 & 1.03 & 7.79110112 & 7.79110112 & 7.79412286 & 7.79839288 \\
\hline 0.2 & 1.05 & 4.06123912 & 4.06123912 & 4.06097156 & 4.06462461 \\
\hline 0.2 & 1.07 & 2.58514629 & 2.58526457 & 2.55870679 & 2.58327072 \\
\hline 0.185 & 1.02 & 11.09392484 & 11.09392487 & 11.09352571 & 11.09955263 \\
\hline 0.185 & 1.03 & 6.36895584 & 6.36895584 & 6.36691545 & 6.37541945 \\
\hline 0.185 & 1.05 & 2.93819009 & 2.93819009 & 2.93903905 & 2.94327857 \\
\hline 0.185 & 1.07 & 1.67103288 & 1.67103288 & 1.64945221 & 1.67268370 \\
\hline
\end{tabular}

For our standard model parameters we now give a representation of optimal strategies via probability levels $M(b), b=K, K+1, \ldots$ which are the minimal allowed ruin probabilities $\alpha$ for which the optimal dividend strategy with value $V(b, \alpha)$ starts dividend payment at $b+1$.

If $\alpha$ is an allowed ruin probability for initial surplus $s_{0}$, then we start dividend payment at $b \geq s_{0}$ with an allowed ruin probability $\alpha(b)=1-(1-\alpha) f(b) / f\left(s_{0}\right)$ when this probability exceeds the critical probability level $M(b)$. Values for $M(b)$ are listed in the following Table 3 . Notice that the allowed ruin probabilities $\alpha(b)$ differ from the running ruin probabilities: they are computed for the initial surplus $s_{0}$, not for the current state $s$. 
Table 3. Critical probability levels.

\begin{tabular}{cccc}
\hline $\boldsymbol{b}$ & $\boldsymbol{M}(\boldsymbol{b})$ & $\boldsymbol{b}$ & $\boldsymbol{M}(\boldsymbol{b})$ \\
\hline 4 & 0.259731506201395 & 15 & 0.183678509907968 \\
\hline 5 & 0.210250720453755 & 16 & 0.183675629162660 \\
\hline 6 & 0.194672114697339 & 17 & 0.183674394994606 \\
\hline 7 & 0.188157745948590 & 18 & 0.183673887714509 \\
\hline 8 & 0.185579934696899 & 19 & 0.183673639474299 \\
\hline 9 & 0.184488131429990 & 20 & 0.183673542359286 \\
\hline 10 & 0.184022232778670 & 21 & 0.183673500613837 \\
\hline 11 & 0.183822880902840 & 22 & 0.183673483500257 \\
\hline 12 & 0.183737493786624 & 23 & 0.183673475435889 \\
\hline 13 & 0.183700929021706 & 24 & 0.183673471845663 \\
\hline 14 & 0.183685869343539 & 25 & 0.183673470498570 \\
\hline
\end{tabular}

Again, we consider only $T=0-$ and $T=1$-payments. We choose $Z$ and $E$ ( $Z$ the number of $T=1$-payments and $E$ of $T=0$-payments at $b$ ) such that the resulting next allowed ruin probability

$$
\alpha(b+1)=1-(1-\alpha(b))(f(b+1) / f(b-1))^{Z}(f(b+1) / f(b))^{E}
$$

still exceeds $M(b)$.

In our example with $s_{0}=1$ and $\alpha=0.2$ we get $b=6$ (since $0.2>M(5)$ ) and the following possible choices for the pairs $(Z, E)$ with corresponding allowed ruin probabilities $\alpha(7)$ :

All other choices lead to a value $\alpha(7)<M(6)$.

For the allowed ruin probabilities the factors leading from barrier $b$ to $b+1$ when exponents $Z$ and $E$ are used at barrier $b$ we obtain:

$$
(f(b+1) / f(b-1))^{Z}(f(b+1) / f(b))^{E} .
$$

The discount factor for dividend payment at $b$ equals

$$
(w(b+1) / f(b-1))^{Z}(f(b+1) / f(b))^{E} .
$$

We include a short example for the calculations of present values of dividend payments for the case that the initial surplus $s_{0}$ is larger than the first barrier $B_{0}$ at which dividends are paid (see Section 3 ). Again, we use the model parameters $p=0.7$ and $r=1 / 1.03$. Our allowed ruin probability $\alpha$ is the one corresponding to $\alpha=0.2$ when $s_{0}=1$. We obtain $b=6$, and for $s_{0}=7, \ldots, 13$ we obtained always the $T=1$-payment as an optimum. For $s_{0}$ above 13 the payment of a lump sum $s_{0}-6$ is always optimal. The critical boundary for $V\left(B_{0}, \alpha\right)$ in (34) is $23.33 \ldots$ The calculations are given in our Maple file.

Finally, we give numerical results for Equations (51) and (52) in our standard example where we have $b=6$ and $Z(6)=E(6)=1$. The present value for $\alpha=0.2$ is 7.79839288. For $E(6)=0$ we obtain

$$
\alpha_{1}=1-(1-\alpha) f(7) / f(6)=0.19878278
$$

and

$$
V\left(s_{0}, \alpha_{1}\right)=P v-\frac{w(7)}{w(6)}\left(P v-V\left(s_{0}, \alpha\right)\right)=7.75057041 .
$$

The values for $Z(6)=0$ and $E(6)=1$ are

$$
\alpha_{1}=1-(1-\alpha) f(7) / f(5)=0.19592814,
$$




$$
V\left(s_{0}, \alpha_{1}\right)=P v-\frac{w(7)}{v(1) w(6)}\left(P v-V\left(s_{0}, \alpha\right)\right)=7.62217520 .
$$

Setting both exponents to zero, $Z(6)=E(6)=0$, then

$$
\alpha_{1}=1-(1-\alpha) f(7)^{2} /(f(5) f(6))=0.193063,
$$

and

$$
V\left(s_{0}, \alpha_{1}\right)=P v-\frac{w(7)}{v(1) w(6)} \frac{w(7)}{w(6)}\left(P v-V\left(s_{0}, \alpha\right)\right)=7.46965095 .
$$

In the MatLab calculations, we observed that the exponents $Z(k), E(k), k \geq 7$, for $V\left(s_{0}, \alpha_{1}\right)$ and $V\left(s_{0}, \alpha\right)$ do not coincide for all $k \geq 7$ due to numerical inaccuracy. But the differences occur for $k \geq 40$ only and have no significant influence on the present values.

\section{Maple File}

Here we enclose a Maple file as well as some MatLab files with which the above calculations were performed. The Maple code can be copied directly at a prompt in Maple. There, the high precision is needed when computation is done for states from $s=0$ to $s=300$. The code computes the maximal present value $V(s, \alpha)$ for dividend strategies with limits $B_{i}-1$. This code is made for possible interaction: the first barrier $b=B_{0}$ has to be chosen manually, small but large enough to get a positive value $e x$.

The Maple code deals with the situation of $p=0.7, r=1 / 1.03, \alpha=0.2$ and $s_{0}=1$. The code also computes the dividend value of a two-barrier dividend strategy with maximized value and limits $L_{i}=B_{i}-1$ as well as with mixed strategies with limits $B_{i}$ as well as $B_{i}-1$. The pure $T=1$-strategy is obtained for $E[i]=0, i \geq 4$, and the values presented above with, e.g., $E[6]=E[11]=E[18]=1$.

\section{Maple code for mixed strategies}

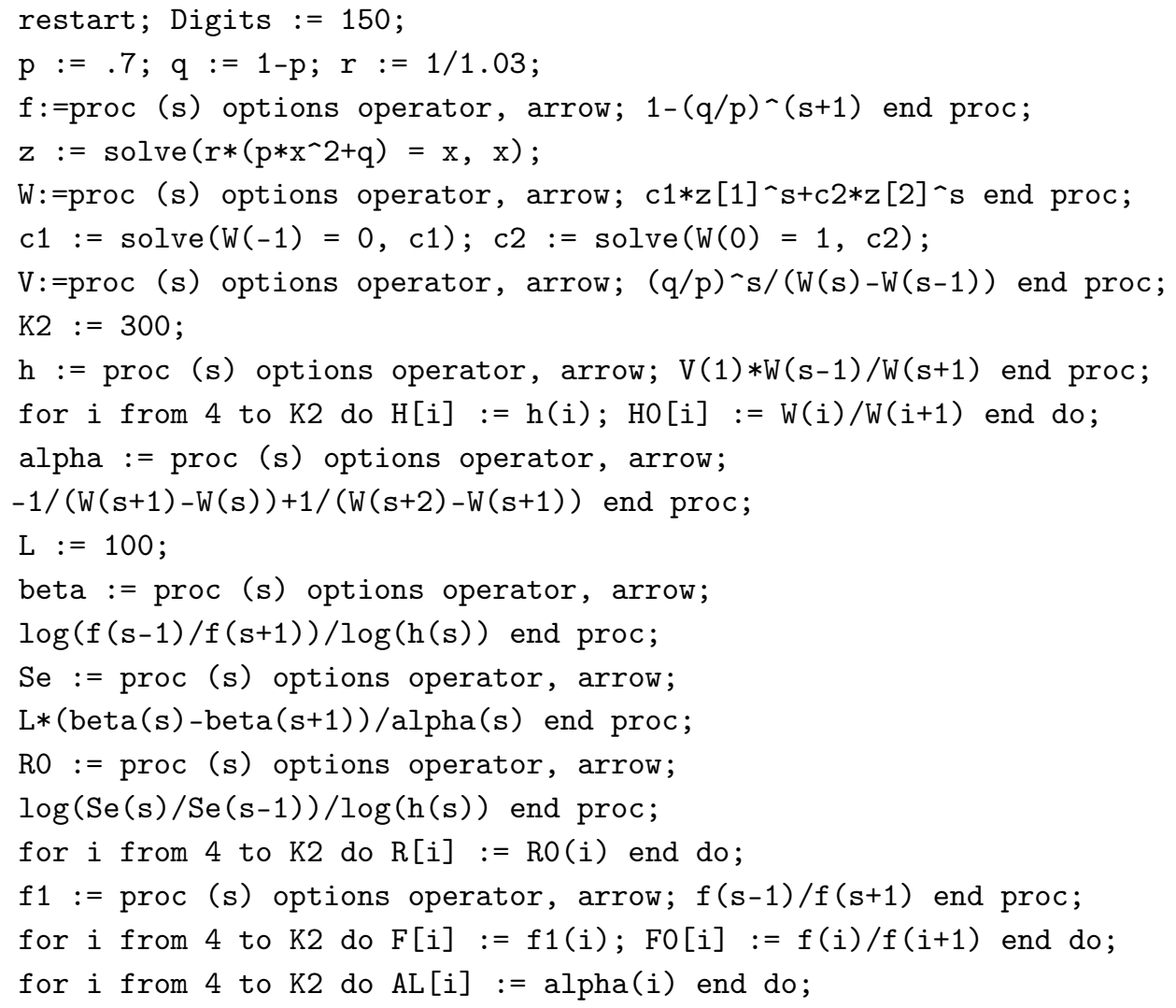




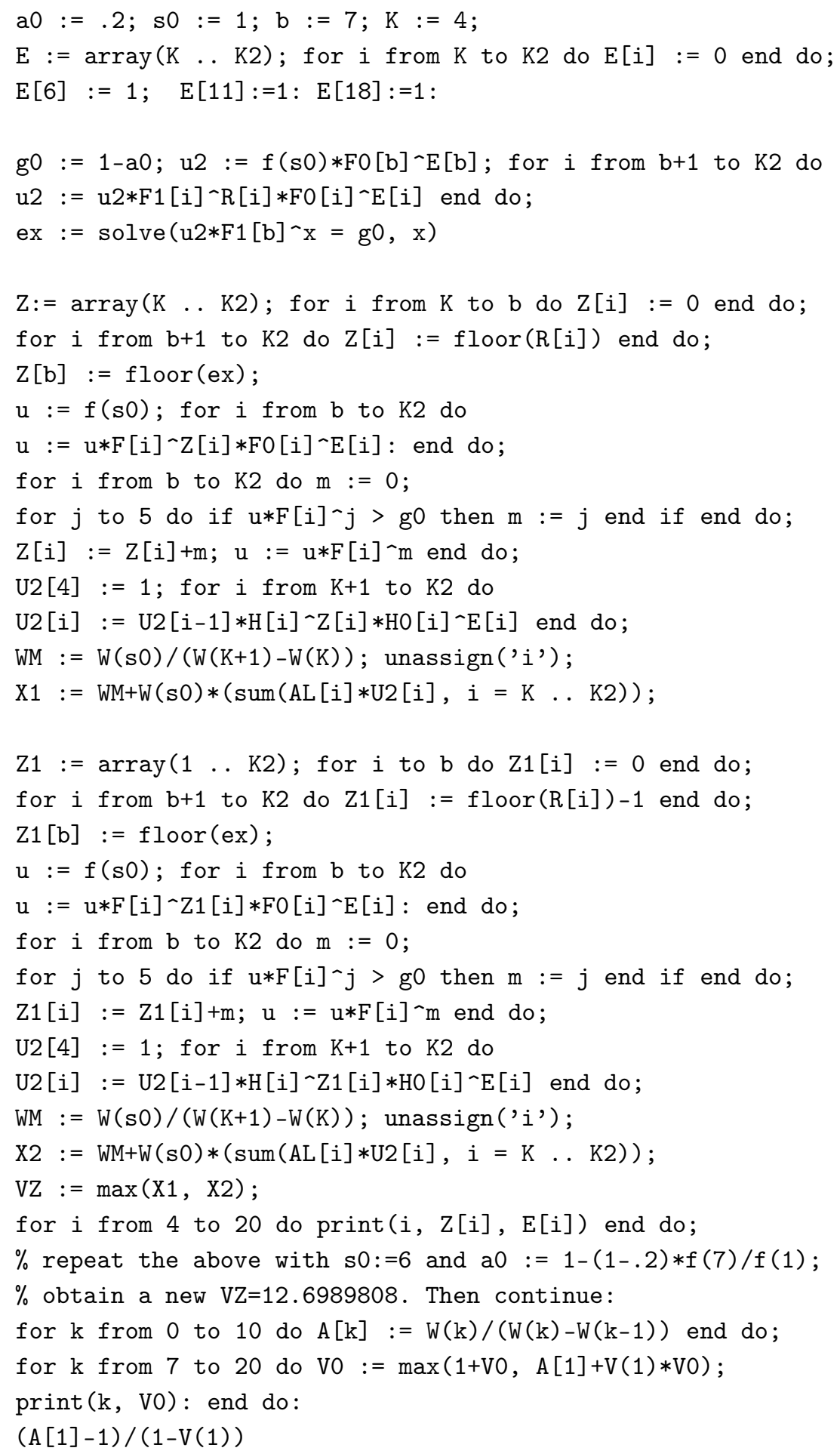

The MatLab codes are easier to use, and despite the lower accuracy of MatLab they provide ten or more valid digits in the dividend values. In short time one can compute a large number of dividend values for different allowed ruin probabilities (e.g., for plots or comparison of methods). The submodules DeFinettiIni.m and DefinettiCalc.m are separated for better readability. The main code is DeFinettiOne.m for the computation of one case for the mixed strategies for $V(s, \alpha)$; it can also serve for the computation of a function for plotting purpose. Slight modifications are needed for the case of pure $T=1$ - payments or pure $T=0$ - payments. Notice that the curves for pure $T=0-$ strategies, $T=1-$ strategies as well as for mixed strategies all look the same, the differences are visible only when the plots are blown up substantially. 
The code Barrier.m produces the probability levels in Table 4. It uses the codes above: e.g., DeFinettiOne.m in which the fixing of $a 0$ must be commented out. In our calculations we saw that the dividend strategies produced discontinuities of $\alpha \rightarrow V(s, \alpha)$ at the probability levels $M(b)$. To eliminate these, we modified the strategies in DeFinettiOne.m: we allow for first dividend payment at $b$ as well as at $b+1$ and take the maximum of the resulting present values.

Table 4. Allowed ruin probabilities.

\begin{tabular}{cc}
\hline$Z, E$ & $\alpha(7)$ \\
\hline 0,1 & 0.1987827794 \\
\hline 0,2 & 0.1975637068 \\
\hline 0,3 & 0.1963427793 \\
\hline 1,0 & 0.1959281420 \\
\hline 1,1 & 0.1947047260 \\
\hline
\end{tabular}

\section{DeFinettiIni.m}

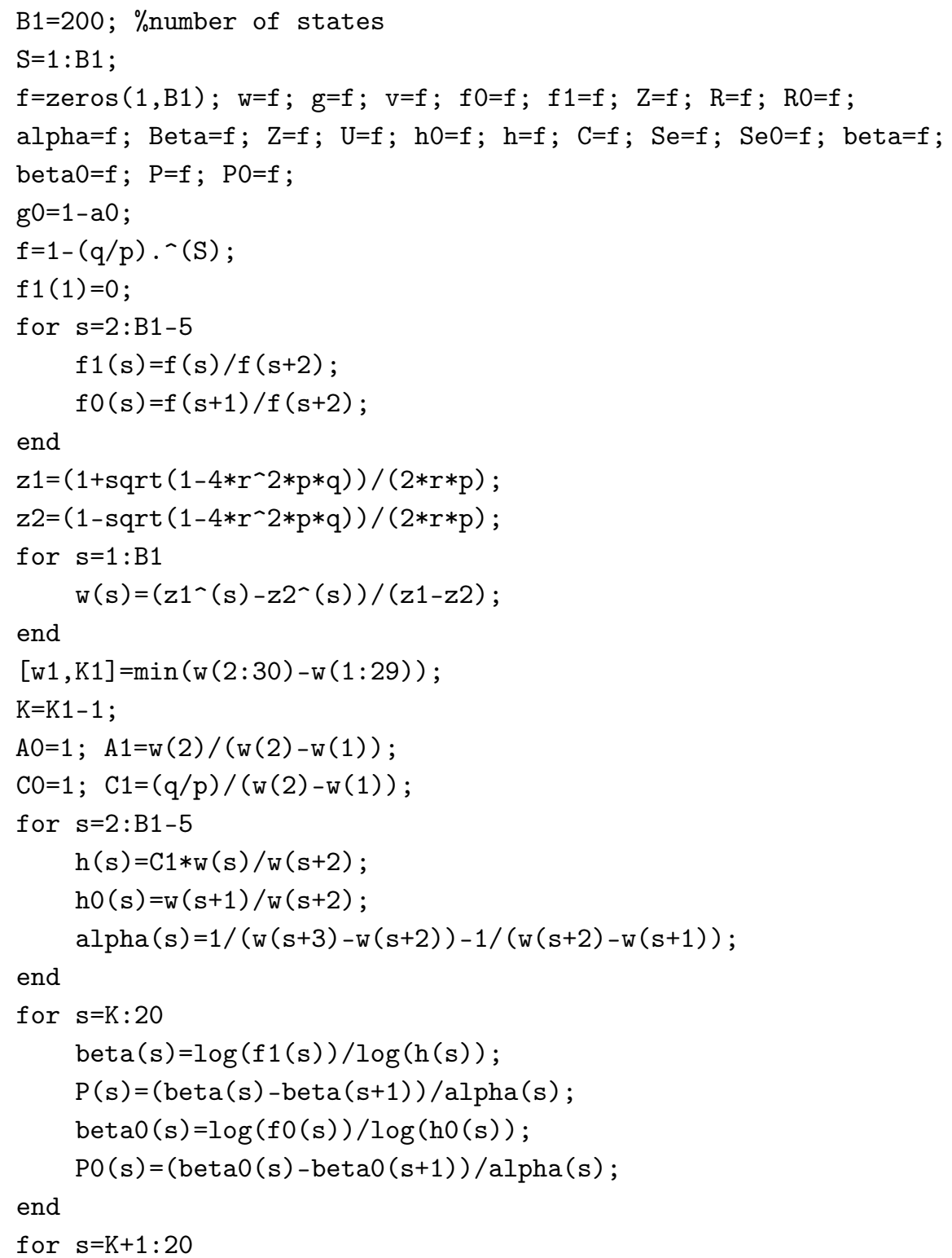




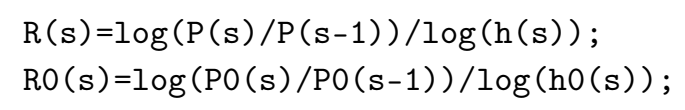

\section{DeFinettiCalc.m}

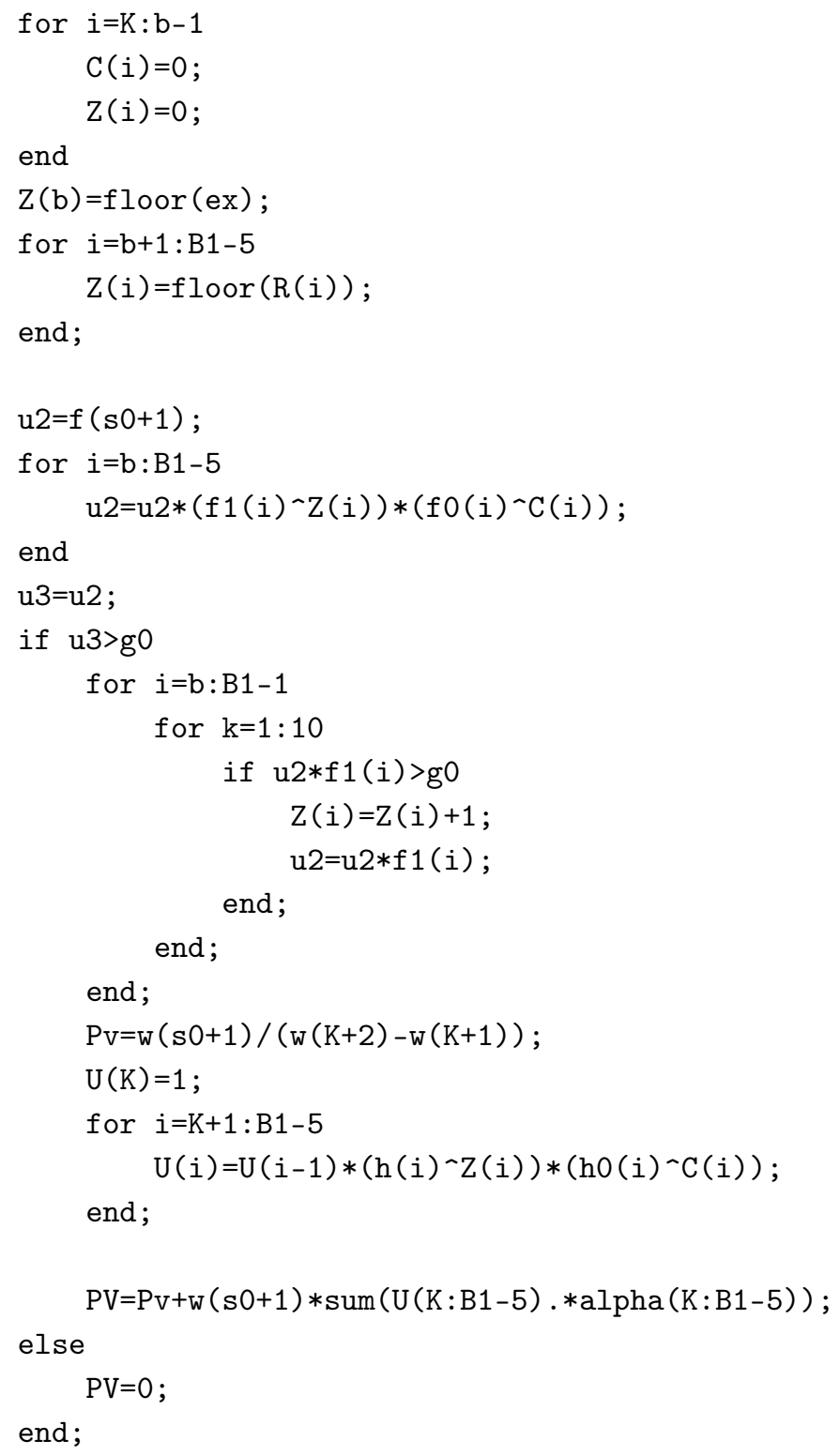




\section{DeFinettiOne.m}

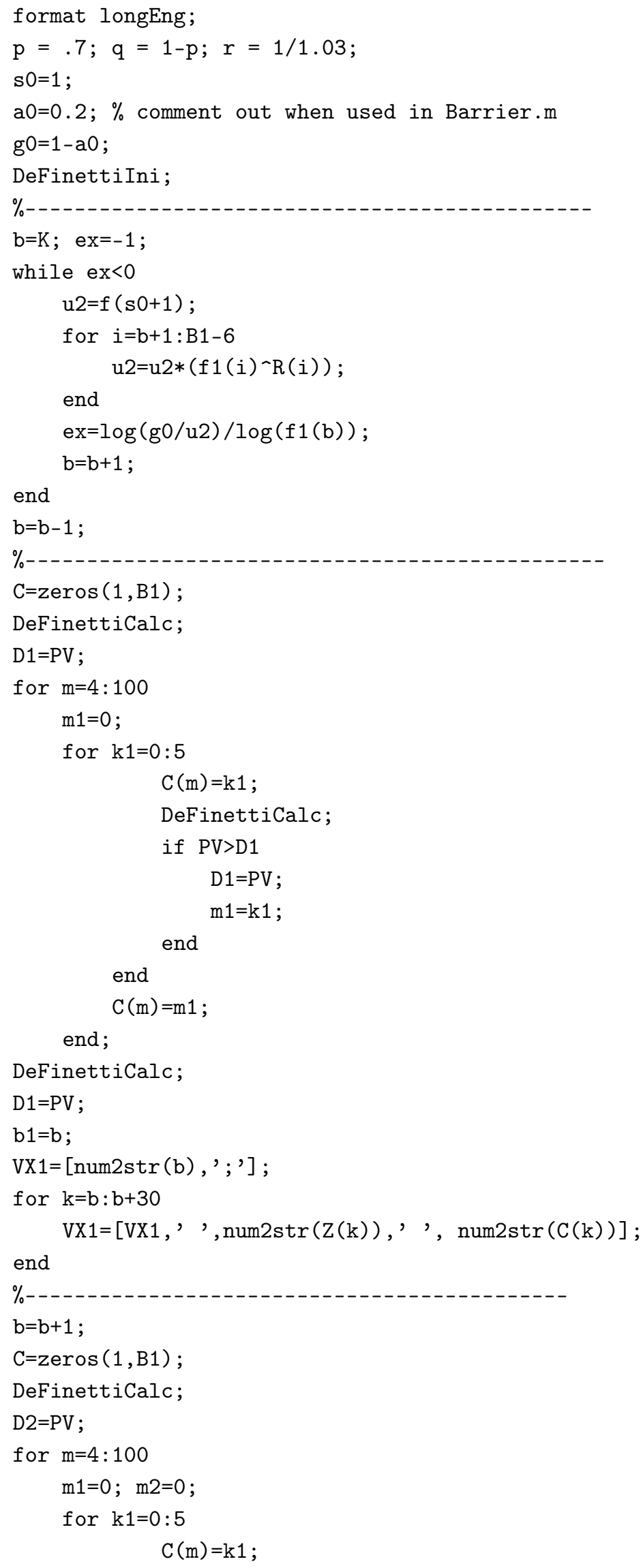




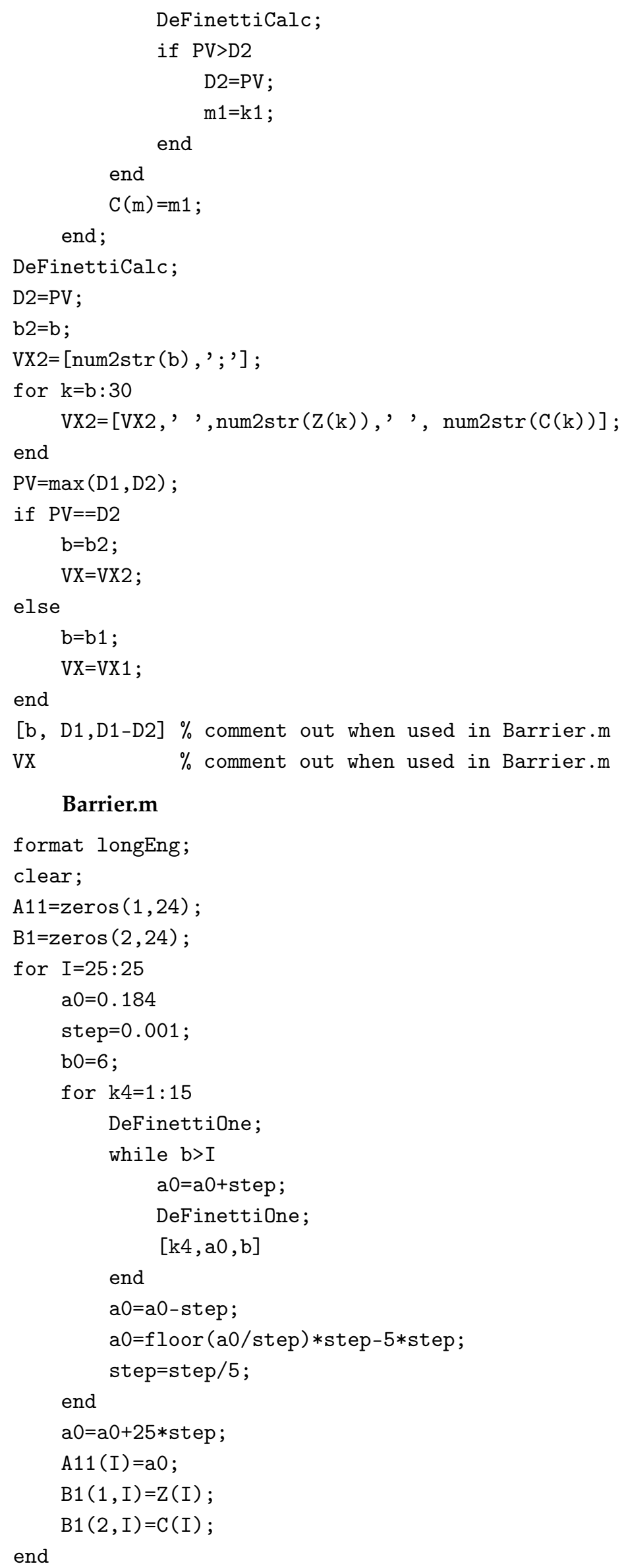

\section{Barrier.m}

format longEng;

clear;

$A 11=\operatorname{zeros}(1,24)$;

$\mathrm{B} 1=\operatorname{zeros}(2,24)$;

for $I=25: 25$

$\mathrm{a} 0=0.184$

step $=0.001$

$\mathrm{b} 0=6$;

for $k 4=1: 15$

DeFinettiOne;

while $b>I$

$\mathrm{a} 0=\mathrm{a} 0+$ step;

DeFinettiOne;

$[\mathrm{k} 4, \mathrm{a} 0, \mathrm{~b}]$

end

$\mathrm{a} 0=\mathrm{a} 0$-step;

$\mathrm{a} 0=\mathrm{fl}$ loor $(\mathrm{a} 0 /$ step $) *$ step- $5 *$ step;

step=step $/ 5$;

end

$\mathrm{a} 0=\mathrm{a} 0+25 *$ step;

$\mathrm{A} 11(\mathrm{I})=\mathrm{a} 0$;

$\mathrm{B} 1(1, \mathrm{I})=\mathrm{Z}(\mathrm{I})$;

$\mathrm{B} 1(2, \mathrm{I})=\mathrm{C}(\mathrm{I})$;

end 
Funding: This research received no external funding.

Conflicts of Interest: The authors declare no conflict of interest.

\section{References}

Albrecher, Hansjoerg, and Reinhold Kainhofer. 2002. Risk theory with a non-linear dividend barrier. Computing 68: 289-311. [CrossRef]

Albrecher, Hansjoerg, and Stefan Thonhauser. 2007. Dividend maximization under consideration of the time value of ruin. Insurance Mathematics and Economics 41: 163-84. [CrossRef]

Albrecher, Hansjoerg, and Stefan Thonhauser. 2009. Optimality results for dividend problems in insurance. RACSAM-Revista de la Real Academia de Ciencias Exactas, Fisicas y Naturales. Serie A. Matematicas 103: 295-320. [CrossRef]

Asmussen, Soeren, and Michael Taksar. 1997. Controlled diffusion models for optimal dividend pay-out. Insurance Mathematics and Economics 20: 1-15. [CrossRef]

Azcue, Pablo, and Nora Muler. 2005. Optimal reinsurance and dividend distribution policies in the Cramér-Lundberg model. Mathematical Finance 15: 261-308. [CrossRef]

Belhaj, Mohamed. 2010. Optimal dividend payments when cash reserves follow a jump-diffusion process. Mathematical Finance 20: 313-25. [CrossRef]

De Finetti, Bruno. 1957. Su un impostazione alternativa della teoria collettiva del rischio. In Transactions of the 15th International Congress of Actuaries, New York and Toronto. Ottawa: International Actuarial Association, pp. 433-443.

Dickson, David, and Steve Drekic. 2008. Optimal dividends under a ruin probability constraint. Annals of Actuarial Science 1: 291-306. [CrossRef]

Gerber, Hans U. 1969. Entscheidungskriterien für den Zusammengesetzten Poisson-Prozess. Ph.D. thesis, ETH Zurich, Zurich, Switzerland.

Gerber, Hans U. 1981. On the probability of ruin in the presence of a linear dividend barrier. Scandinavian Actuarial Journal 1981: 105-15. [CrossRef]

Gerber, Hans U., Elias SW Shiu, and Hailiang Yang. 2010. An elementary approach to discrete models of dividend strategies. Insurance Mathematics and Economics 46: 109-16. [CrossRef]

Hipp, Christian. 2003. Optimal dividend payment under a ruin constraint: discrete time and state space. Blätter der DGVFM 26: 255-64. [CrossRef]

Hipp, Christian. 2018. Company value with ruin constraint in a discrete model. Risks 6: 73. [CrossRef]

Hipp, Christian. 2019. Dividend payment with ruin constraint. In Risk and Stochastics, Ragnar Norberg. Edited by P. Barrieu. Hackensack: World Scientific, pp. 1-22, ISBN 978-17-86341-945.

Kulenko, Natalie, and Hanspeter Schmidli. 2008. Optimal dividend strategies in a Cramér-Lundberg model with capital injections. Insurance Mathematics and Economics 43: 270-78. [CrossRef]

Loeffen, Ronnie. 2008. On optimality of the barrier strategy in De Finetti's dividend problem for spectrally negative Lévy processes. The Annals of Applied Probability 18: 1669-80. [CrossRef]

Schmidli, Hanspeter. 2007. Stochastic Control in Insurance. Heidelberg: Springer, ISBN 978-1-84800-003-2.

Sotomayor, Luz R., and Abel Cadenillas. 2011. Classical and singular stochastic control for the optimal dividend policy when there is regime switching. Insurance Mathematics and Economics 48: 344-54. 